\title{
A New Remote Monitoring System Application in Laser Power Based on LabVIEW
}

\author{
Gaoqiang Liu, Weilin Wu, Mingyu Liu, Kun Zhang and Guannan Chen ${ }^{\text {a }}$ \\ Key Laboratory of Optoelectronic Science and Technology for Medicine, Ministry of Education \\ Fujian Normal University \\ Fuzhou, 350007, China
}

\begin{abstract}
In this paper, a new remote monitoring system based on LabVIEW was proposed to measure laser power automatically and remotely. This system consists of four basic components: an DH-JG2 optical power meter, a NI-USB 6008 data acquisition card, a personal computer (PC), and HP laserJet 1020 Plus printer. Since power output of laser is generally so unstable that abnormal work situation could not retroaction to inspectors right away, new system was designed to solve this problem. The detection system realized function of remote control by TCP protocol and mobile phone. Laser power curve that is measured by detection system demonstrated that the design has a good performance in real-time detection and operability.
\end{abstract}

\section{Introduction}

Laser is the important component of technologies in the field of communication, manufacturing, as well as optical experiment because of its characteristics including high brightness, good monocolor, good coherence as well as good direction. However, as far application, one of the main problems is that its output power is not stable and the laser is easy to be destroyed because of transient circuit and voltage peak[1]. Lots of power meters were invented for measurement in the process of laser manufacture last decades. With the rapid development of the computer technology, manual operation desktop machines have the disadvantages of low efficiency, inconvenient data analysis and storage in the quality of laser testing[2]. Therefore, software-based automated testing system design is imperative. Measurement system of laser power in $\mathrm{VC}++[3]$, the software of optical power measurement and calibration and instrument control system based on LabVIEW have overcame the lack of the manual operation desktop machines, but they remains to be more automatical, and errors in the testing process can't be managed when failed in working in time[4].

With the rapid development of the computer technology and the software technology, there are more and more kinds of software development platforms and instrument functions which can be achieved by computers and related software NI-LabVIEW[5]. LabVIEW produced by National Instruments is the most widely used as virtual instrumentation system. It has the development and debugging tools of traditional programming language. The greatest feature of LabVIEW is the graphical programming language, which is easy to grasp, understand develop a friendly, realistic user interface. Furthermore, it has the powerful signal processing ability, and integrates almost all of the features of hardware communications such as data acquisition card. Virtual instrument is an individual computer testing equipment that has a virtual instrument panel. It brings a revolutionary change in the field of automatic testing system. To a testing platform, LabVIEW includes the storage functions of data collection, processing and

\footnotetext{
$\overline{{ }^{\mathrm{a}} \text { Corresponding author:edado@fjnu.edu.cn }}$
} 
analysis. To meet the needs of various practical applications toolbox, it can use dynamic link (DLL) technology to users of the virtual instrument software to link to them. use links embedded object (OLE) technology will be embedded in other virtual instruments. LabVIEW offers a numbers of source-level instrument drivers, including DAQ, GPIB, PXI, VXI, RS232 as well as USB, users can choose their own hardware drivers according their own needs[6].

In this paper, a remote monitoring system in laser power based on Labview was designed and implemented. The system used optical power meter DH-JG2 as monitoring laser power component produced by DaHeng. NI-USB 6008 data acquisition card was utilized to collect the power data from power meter. A electrical energy supply system HSPY_36_03 produced by HSPY was employed for turning on or off the laser, Also HP laserJet 1020 Plus as the output device of the test report. Besides, using the real time software LabVIEW displayed and analyzed the power data, and a mobile phone was used to remotely monitor.

\section{System composition}

System design framework is shown in fig. 1, from which we can see the system consisting of Laser, power meter, data acquisition card, printer, PC machine, electrical energy supply system as well as mobile phone. In which, DH-JG2 is the power meter, responsible for collecting the actual laser power data, and outputting voltage signals. And the USB 6008 is a data acquisition card produced by NI(National Instrument), can collect voltage signals from DH-JG2, convert the signals into digital signals and send the digital signals to the PC machine by USB bus[7]. On the one hand, PC machine displays and analyses the power data, and generates a test report and sends it to the HP laserJet 1020 plus and then the report would be printed by the printer. In addition, the system can easily to turn off laser through sending the codes to HSPY_36_03 and then laser will stop working until the electrical energy supply down to zero. A mobile phone is needed in remotely monitor system.

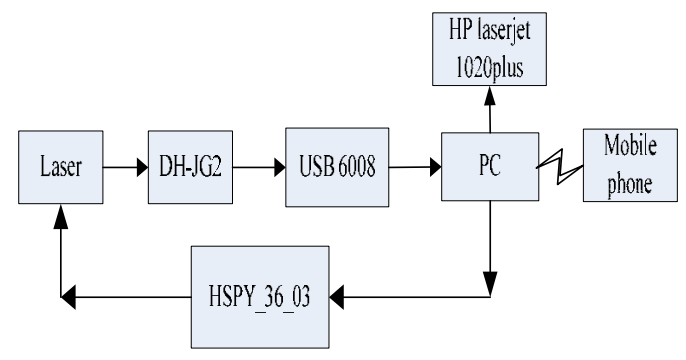

Figure 1. Framework map of system design

\section{LabVIEW Program}

The LabVIEW has been used to acquire the laser power data obtained from power meter, and display the raw data. After data of laser's power waveform acquisition, the data are sent to Express Virtual Instruments for signal processing. To evaluate the quality of the laser, the program will find the maximum, minimum as well as average value from the processed signal in order to calculate the stability of laser power. After that, the system needs to generate the test report and output it by printer. Virtual Instrument (VI) provides Report Generation tools and thus the test report is produced.

\subsection{Data Acquisition}

In this system, setting the measurement time interval and the total measurement time are required. After that we can easily read out the measurement results of fixed interval. Comapared to the traditional measurement of power stability that is measured by inspectors and need recorded measurements within 24 hours continuously, this system



Figure 2. The program of data acquisition

can save labor force. The block program of data acquisition is shown as Fig. 2, which includes DAQmx 
acquisition functions, Mean filter and measurement count. When the power meter and the software connect to the DAQ card[8], we can measure the output the laser power automatically. And the data real-time displayed on the wave diagram, we can realize the trend of power. Fig. 3 shows the front panel structure of the data acquisition, in which the results of Measurement 4.28 is just the measured output power[9].

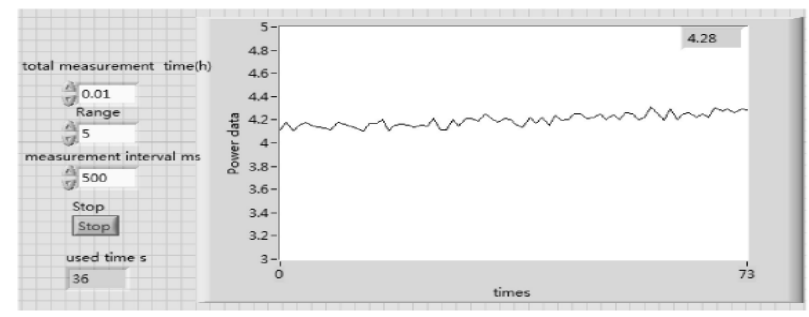

Figure 3. The front panel structure of the data acquisition

\subsection{Data Analysis}

The data analysis includes displaying real-time laser power data and extracting the data of the average, maximum, minimum, peak-peak and the stability value. The stability value was calculated in Equation 1.

$$
S_{w}=\frac{\left(P_{\max }-P_{\min }\right)}{P_{a}} \times 100 \%
$$

Where, $S_{w}$ is the stability of the laser power, and the $P_{\max }, P_{\min }$, and $P_{a}$ represent maximum, minimum and average value of the laser power, respectively. The Statistics function was used to get the average, maximum, minimum, peak-peak value of the power data[10]. Fig. 4 and Fig. 5 show the front panel procedure and the block diagram procedure of LabVIEW of the laser's power data analysis.

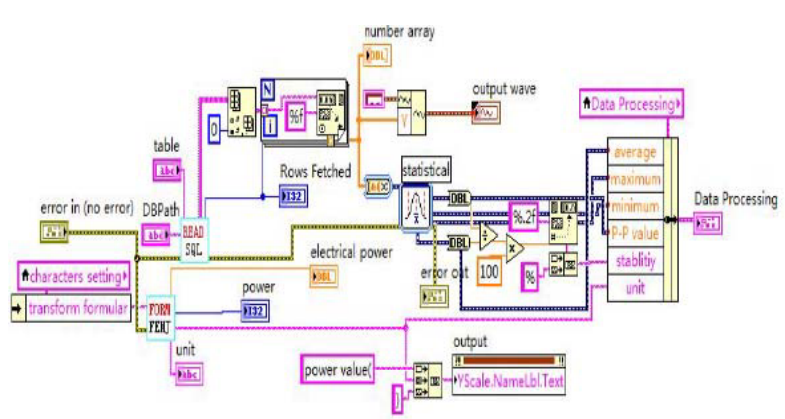

Figure 4. The program of data analysis

\subsection{Application and testing}

Fig. 6 shows the working model of the system. Before start testing, the Parameters Setting part that is the details about the laser and the test settings including testing time, collect interval, the type of power meter etc. should be

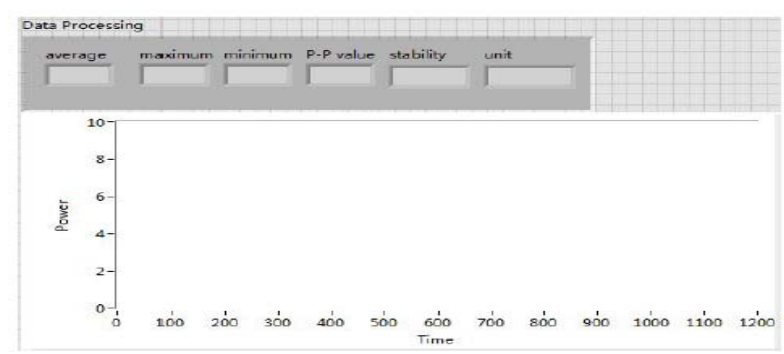

Figure 5. The block diagram procedure of LabVIEW

filled in. When we start collecting, the power data will be real-timely displayed in the Measurement result block like the blue swash. After collecting, the data analysis results block like the blue swash. After collecting, the data analysis results will be shown in the Data Processing block. In order to output the report, we use Report Generation tools. And the report is shown in Fig. 7.

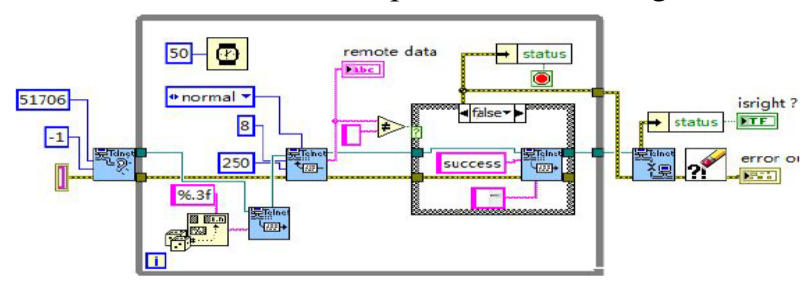

Figure 6. The working model of the system



Figure 7. The remote client interface

\section{Remote monitor}

Remote monitoring of laser power is implemented by TCP communications. LabVIEW can achieve TCP communications by using the published TCP module. In this paper, we designed server mode by LabVIEW in PC machine and client mode by android that used in a mobile phone. The first part of the program is the server mode including data acquisition, data analysis, and the report generation laser controlling worked in the server model. Besides, the second part is the client model consisting of the management, display and remote monitor implemented by the mobile phone.

In server, using Telnet Create Listen receives the 
connection request of the client. The Telnet tools is based on TCP protocol .The establishment of TCP connection, using Telnet Read function nodes to receive the data which sent by the client and the analyze and process, using Telnet Write function nodes send over data and 'success' to the client. To send double-type data, the procedures first change the double type data into type of characters by the use of Format value node function, and then send to the network. The process diagram was shown in Fig. 8.

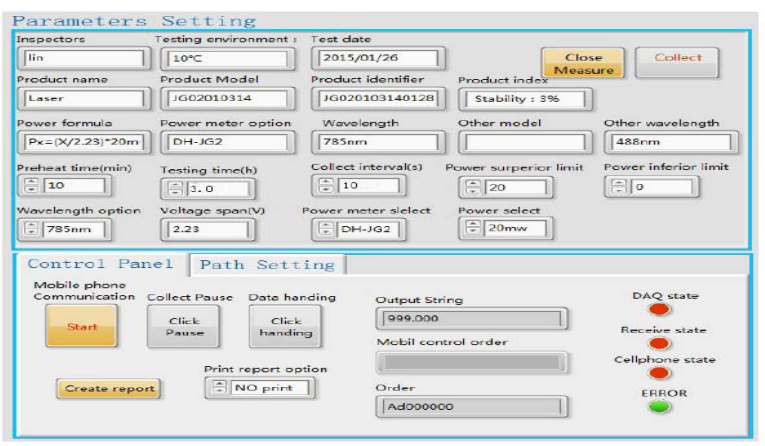

Figure 8. The parameter setting and control interface

In client, the procedure was mainly built by using the Java language in development platform of Eclipse. We can directly realize trend of the laser power data. If the laser works disorderedly, we can send the code that is 'Ss000000' to server model and turn off the laser. In addition, there are many codes with different functions, like as 'Rs000000' means restarting the laser; 'Sc000000' means startting the system to collect the laser power; 'Ar000000' means analyzing the laser power data and printing data etc. By this system, inspectors can conveniently get the working state of laser every second. And the Fig. 9 shows the remote monitoring in mobile phone.

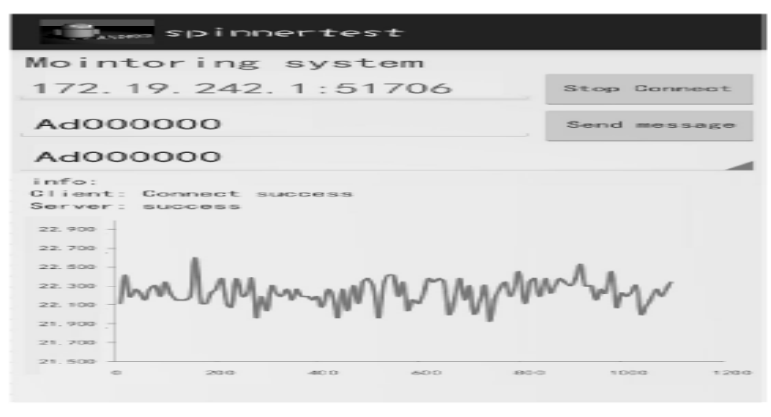

Figure 9. The client synchronization interface

\section{Summary}

In this paper, a new remote monitoring system based on LabVIEW was proposed to measure laser power automatically and remotely. LabVIEW software development platform, combined with data acquisition cards and TCP/IP protocol was used to achieve the power data acquisition, the process of remote monitoring, the data analysis, and report generation. Compared with the traditional data acquisition and processing system, it has the advantages of a cost-effective, high applicability and easy operation. Furthermore, it can make the users using a mobile phone as a remote monitor more convenient. LabVIEW is a powerful front-end design tool for testing system and the monitor system could be used potentially in laser power testing.

\section{Acknowledgement}

This work is supported by the National Natural Science Foundation of China (Nos. 81101110), the Science and Technology Project of Fujian Province (Nos.2015J01300), and Fujian Normal University foundation for excellent young teachers (No. fjsdjk2012058).

\section{References}

1. Swain N K, Anderson J, Singh A, et al. Proceedings. IEEE. 2003, pp. 1-6.

2. Stankovic, L, Signal Processing, vol. 44(1996).

3. Rapuano, S, Zoino, F, IEEE T INSTRUM MEAS, vol. 55, Oct. 2006 , pp. $1757-1766$.

4. Vargas, H. Sanchez-Moreno, J. Dormido, S. Salzmann, C. Gillet, D. Esquembre, F, COMPUT SCI ENG, vol. 11, May.2009 , pp.36,doi: 10.1109/MCSE.2009.61

5. Pan $\mathrm{Yu}, \mathrm{Wu}$ Guoan, Xu Qinfen, Huazhong Univ. of Sci. \& Tech. (Nature Science Edition), vol. 32, Dec. 2004.

6. Ballesteros, J. Fernandez Palop, J. I. Hernandez, M. A, REV SCI INSTRUM, vol. 75, Jan.2004 , pp.90 93.

7. X-Series Programmer's Guide. USA Agilent Technologies, Inc. October 2009.

8. N1911A/1912A P-Series Power Meters Service Guide, USA Agilent, Inc. December 2010.

9. HP 53131A/132A $225 \mathrm{MHz}$ Universal Counter. USA: Hewlett- Packard Company. 1999.

10. GY/T 229.2-2008, Academy of Broadcasting Planning, SARFT. February 2008. 